\title{
Disfunción Endotelial en el personal naval con obesidad en actividad militar
}

\author{
Oscar Luis Alvarez Franco ${ }^{1, a, b}$ \\ RESUMEN
}

Obj etivo: Establecer el nivel de disfunción endotelial en el personal naval con obesidad en actividad militar de la Marina de Guerra del Perú, relacionando además el grado de disfunción endotelial con factores modificables (dieta y actividad física), no modificables (antecedentes familiares) y su estratificación cardiovascular.

Material y Métodos: La función endotelial fue analizada por doppler vascular donde se evaluó el flujo mediado por dilatación en la arteria braquial, de pacientes con obesidad tipo I, II y III, en reposo, analizando su relación con variables como actividad física, tipo de dieta, antecedentes familiares, edad y género; aplicando parámetros estadísticos (chi-cuadrado y análisis de regresión) con SPSS. Estudio de tipo descriptivo, prospectivo y de corte transversal.

Resultados: Se encontró un total de $43.4 \%$ de disfunción endotelial en todos los grupos por edades, de los cuales un $49.3 \%$ correspondía al sexo masculino. De la totalidad de 173 pacientes, el $56 \%$ realizaban ejercicios menos de una hora y recibían dietas mayores de 1800 kcal un total de 113 pacientes encontrándose presencia de disfunción endotelial en el 54\% de pacientes de este grupo; el 65\% de pacientes con antecedentes familiares presentaron $\mathrm{DE}$, siendo ésta una relación estadísticamente significativa $(p<0.05)$ así como también lo fue para la evaluación de edad, sexo y actividad física; siendo la dieta un parámetro estadísticamente no significativo en el análisis de regresión. Conclusión: Los pacientes obesos evaluados presentaron una alta incidencia de disfuncion endotelial, independientemente de la variable asociada a la que estuviera relacionada, puesto que la obesidad tiene una relación directa con la alteración de la función endotelial sin la presencia de enfermedad cardiovascular preexistente ni el acondicionamiento militar previo. Se sugiere para mejorar la alteración endotelial ejercicios que contemplen rutinas mayores a una (01) hora, supervisadas por personal calificado. La dieta, según los resultados del estudio juega un papel importante pero no determinante en la influencia de disfunción endotelial. De forma secundaria la población obesa tiene una afinidad y adherencia a programas de control de sobrepeso y obesidad, y nutrición, sistema que permite la realización de distintas actividades como el reacondicionamiento físico en pacientes de alto riesgo, sin embargo vemos una relación inversa a estos programas por parte de la institución, con el aumento de condiciones laborables sedentarias que no favorecen a las rutinas de ejercicio propuestas. Por último, la evaluación del flujo mediado por dilatación es un marcador de enfermedad cardiovascular, que debe ser considerado dentro de las técnicas de selección para control y seguimiento, pero sobretodo estratificación del paciente obeso sin enfermedad cardiovascular. (Horiz Med 2015; 15(4): 33-43)

Palabras clave: Disfunción endotelial, obesidad, Índice de masa corporal, flujo mediado por dilatación. (Fuente: DeCS BIREME).

\section{Endothelial dysfunction in naval personnel in military activity with obesity}

\section{ABSTRACT}

Objective: To establish the level of endothelial dysfunction in naval personnel in military activity with obesity of the Navy of Peru, also relating the degree of endothelial dysfunction with modifiable factors (diet and physical activity), unchangeable factors (family history) and cardiovascular stratification.

Material and Methods: The endothelial function was analyzed by vascular Doppler, where the flow-mediated dilation in the brachial artery of patients with type I, II and III obesity, at rest was assessed by analyzing its relationship with variables such as physical activity, type of diet, family history, age and gender; applying statistical parameters (chi-square and regression analysis) with SPSS. Descriptive, prospective and cross-sectional study.

Results: A total of $43.4 \%$ of endothelial dysfunction was found in all age groups, of which $49.3 \%$ were male. Of the total 173 patients, $56 \%$ performed exercises less than an hour and a total of 113 patients received over $1800 \mathrm{kcal}$ diet, endothelial dysfunction was found in $54 \%$ of patients in this group; $65 \%$ of patients had a positive family history, this being a statistically significant relationship ( $p<0.05)$ as well as age, sex and physical activity; diet being not statistically significant in the regression analysis parameter.

Conclusion: The obese patients evaluated presented a high incidence of endothelial dysfunction, regardless of the associated variable with which it was related, because obesity is directly related to the impairment of endothelial function without the presence of pre-existing cardiovascular disease or previous military conditioning. It is suggested, to improve the endothelial alteration, exercise routines of more than one (01) hour, supervised by qualified personnel.Diet, according to the survey results, play an important but not decisive influence on the role of endothelial dysfunction. Secondarily the obese population has an affinity and adherence to monitoring programs of overweight and obesity, and nutrition system that allows the realization of different activities such as physical reconditioning in patients at high risk, however we see an inverse relationship to these programs by the institution, with increased sedentary working conditions that do not favor the proposed exercise routines. Finally, assessment of flow-mediated dilatation is a marker for cardiovascular disease, which must be considered in the selection techniques for control and monitoring, but mostly stratification of the obese patient without cardiovascular disease. (Horiz Med 2015; 15(4): 33-43)

Key words: endothelial dysfunction, obesity, body mass index, flow-mediated dilation. (Source: MeSH NLM).

\footnotetext{
Médico Cirujano

Especialidad Cardiología

b Centro Médico Naval "Cirujano Mayor Santiago Távara - CMST"- Marina de Guerra del Perú
} 


\section{INTRODUCCIÓN}

Durante los últimos años se ha visto un incremento de distintas patologías en la población militar activa, con una gran incidencia de problemas de sobrepeso y obesidad, además de la mayor prevalencia de enfermedad cardiovascular y metabólica en dichos pacientes.

Esta situación se ve reflejada en los hallazgos del Servicio de Medicina Preventiva del Centro Médico Naval "Cirujano Mayor Santiago Távara (CMST)" donde se reportó un incremento del $6 \%$ de obesidad respecto al año pasado a través de los controles preventivos anuales realizados en la Unidad de Capacidad Psicofísica, situación preocupante por el incremento de una patología no acorde al sistema de preparación y acondicionamiento físico de unidades operativas y administrativas de la Marina de Guerra del Perú, y por el aumento futuro de enfermedades como Hipertensión Arterial, Dislipidemia, Diabetes Mellitus tipo 2, Enfermedad Coronaria y Enfermedad Arterial Periférica entre otras (1).

Por esta razón, se busca evaluar la problemática por parte de nuestra Institución a cargo del Servicio de Cardiología del Centro Médico Naval - CMST y en conj unto con sus diferentes unidades y servicios, las que regidas por la Dirección de Salud de la Marina velan por el establecimiento de las políticas preventivas en salud y recuperativas, además de plantear los distintos protocolos de acción con el fin de evitar el daño a corto, mediano y largo plazo del personal militar, especialmente en los que se encuentran en pleno desempeño de su actividad.

Considerando esta situación, el área médica de la Institución plantea distintos programas de reacondicionamiento físico y control metabólico nutricional a aquellos quienes son diagnosticados de sobrepeso u obesidad por valoración del Índice de Masa Corporal (IMC) (2), no valorando las condiciones reales de enfermedad cardiovascular por no contar con otros métodos de evaluación más exactos y demostrables, siendo muy importante el precisar el riesgo real de enfermedad y el manejo preventivo, con el fin de disminuir la evolución de las distintas otras enfermedades cardiometabólicas, evitando mayor riesgo de morbimortalidad, además de optimizar los gastos institucionales en el manej 0 preventivo primario, con la disminución de procesos recuperativos y manejo de complicaciones, siendo siempre limitado el presupuesto del Estado.

Esta es una razón estratégica y de vital importancia para plantear el uso de métodos diagnósticos de bajo costo que permitan establecer el nivel de enfermedad cardiovascular, en forma efectiva y precoz. $Y$ es en este sentido, que al enfrentar diariamente las enfermedades cardiovasculares, se plantean nuevas estrategias preventivas.

Por estas razones planteamos este estudio, con el fin de conocer el nivel de disfunción endotelial en el personal militar con obesidad, valorando el índice de masa corporal y los niveles de obesidad en la población militar, además de establecer la relación entre el grado de disfunción endotelial y la obesidad, para estratificación cardiovascular del paciente obeso y la relación que tiene la dieta y la actividad física como determinante de menor disfunción endotelial en los pacientes obesos.

Es relevante la realización de esta investigación en esta Institución, para valorar el nivel de disfunción endotelial en un grupo poblacional tan importante y de alto riesgo como son los pacientes obesos, debido a que los mecanismos de tratamiento y control no están basados en una estratificación real del riesgo cardiovascular, sólo por un índice de masa corporal que lleva a considerar el manejo del peso como único parámetro de seguimiento, siendo necesario considerar muchas veces tratamientos asociados a la dieta (3) y los ejercicios (4) con el fin de disminuir el nivel de disfunción endotelial, evitando o retrasando enfermedades asociadas a este problema, como la Hipertensión Arterial, Diabetes Mellitus, etc; por lo tanto mejorando la salud cardiovascular en pacientes en la plenitud de su ejercicio profesional como militares y asegurando un balance adecuado en los costos/ beneficios de tratamientos preventivos para la Institución dado que este método de diagnóstico (Evaluación Doppler) es un método práctico y sencillo con la capacidad de realizarse en el Servicio de Ecocardiografía del Centro Médico Naval $(5,6)$. 


\section{MATERIAL Y MÉTODOS}

La investigación a realizar es de tipo cuantitativo, descriptivo y de corte transversal. La población considerada parte del estudio será todo el personal de Marina en actividad que pase su examen psicofísico anual, durante el 01 de marzo al 30 de abril 2015.

Al evaluar nuestra población encontramos un total de 1432 personas con diagnóstico de sobrepeso y obesidad, de los cuales en grado de obesidad I, II y III se encontraron 468 individuos.

A ellos se aplicaron criterios de inclusión y exclusión con el fin de tener una población ideal para la evaluación de la disfunción endotelial, sin mayores condicionamientos y factores que puedan desvirtuar los resultados, considerando que la obesidad se asocia a enfermedad cardiovascular. Se tomaron los siguientes criterios (7-13).

Criterios de Inclusión

1. Índice de Masa Corporal mayor o igual a 30.0 $\mathrm{Kg} / \mathrm{m} 2$.

2. Entre 25 - 59 años.

3. Reposo físico absoluto en las 24 horas anteriores a la realización de la evaluación.

4. Aceptación de participar en el estudio mediante la firma del consentimiento informado.

Criterios de Exclusión

1. Consumo de cualquier número de cigarrillos en los últimos 6 meses.

2. No padecer de enfermedades crónicas, entre ellas:

a) Hipertensión arterial (02 tomas de presión arterial $>130 / 80 \mathrm{mmHg}$ en ambos brazos y con $5 \mathrm{~min}$ de reposo).

b) Diabetes mellitus (glicemia en ayunas $>126 \mathrm{mg}$ / dl).

c) Dislipidemia (valor de triglicéridos $>150 \mathrm{mgr} / \mathrm{dl}$ $\mathrm{y} /$ o colesterol $>200 \mathrm{mg} / \mathrm{dl}$ en sangre).

d) Insuficiencia renal crónica (valor de creatinina $>1.5 \mathrm{mg}$ en sangre).

e) Enfermedad cardiaca o vascular (por datos de anamnesis y revisión de historia clínica). f) Infección por VIH - SIDA (por anamnesis y revisión de historia clínica).

3. Consumo de alcohol (no ingesta de alcohol 07 días antes de la prueba).

4. Síndromes febriles o cuadros infecciosos.

5. Consumo de fármacos (antineoplásicos, glucocorticoides, agentes psicoactivos, broncodilatadores). En mujeres uso de anticonceptivos y estar fuera de 6to al 15to día del ciclo menstrual.

De este grupo, aplicando los criterios de inclusión para el estudio, se obtuvieron 316 sujetos que cumplían las características básicas, entre hombres y mujeres.

En ellos se aplicó la siguiente estructura de muestreo: el tamaño de la muestra se determinó utilizando la fórmula de cálculo de porcentajes para investigación descriptiva, aceptando un error estándar hasta $5 \%$ (0.05), con un nivel de confianza del $95 \%$, asumiendo una $\mathrm{p}=0.5$ y $q=0.5$ y una precisión del $5 \%$. Población total $\mathrm{N}=316$, siendo por lo tanto el valor de $\mathrm{n}=173$.

Como criterios de diagnóstico de disfunción endotelial se consideró la presencia de una dilatación menor del $5 \%$ del diámetro de la arteria humeral post oclusión (con manguito de presión) por un tiempo de 5 minutos $(14,15)$.

\section{RESULTADOS}

La distribución de la edad en los pacientes se estableció por rango de edad, considerando grupos específicos por edad (relacionados a grupos etarios en la Escala de Framingham por factor de riesgo cardiovascular) siendo un total de siete (07) grupos.

En total tenemos $43.4 \%$ de disfunción endotelial en todo los grupos por edades, alto considerando que el promedio de edad es de 37,3 años.

Se observa que según la edad del paciente, la relación se hace directamente proporcional, obteniendo un nivel mayor de casos de disfunción endotelial en los grupos de mayor edad. Realizando un análisis multivariable de todos los sujetos de nuestro 
estudio encontramos una relación estadísticamente significativa $(\mathrm{p}<0.05)$ entre la edad y la presencia de disfunción endotelial independientemente de otros factores.

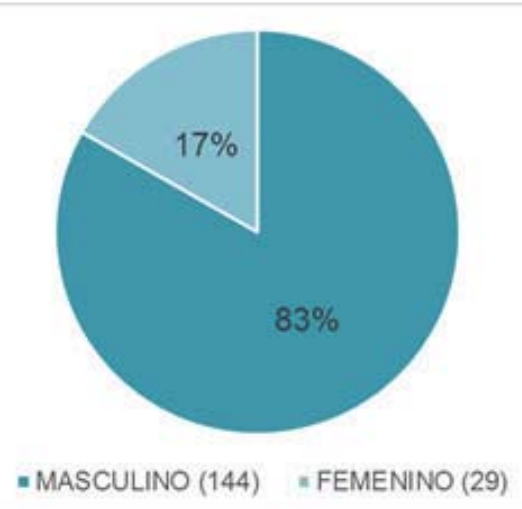

Figura 1. Distribución por sexo en el personal militar con obesidad

Por otra parte, de la totalidad de pacientes de la muestra, se encontró que sólo el $17 \%$ fueron mujeres, una situación particular por la cantidad de personal femenino con la que cuenta la institución militar, pero sin ser ajena a la presencia de obesidad.

En el grupo de las mujeres, se presentó un patrón de disfunción endotelial en un $13.7 \%$ y en el grupo de los hombres un $49.3 \%$; esto en particular determina una característica que evaluar por la condición de menor prevalencia en pacientes de sexo femenino.

En nuestra muestra encontramos una relación significativa $(p<0.05)$ para ambos grupos, pero existiendo una menor condición de disfunción endotelial en mujeres de menor edad respecto al grupo de hombres de menor edad.

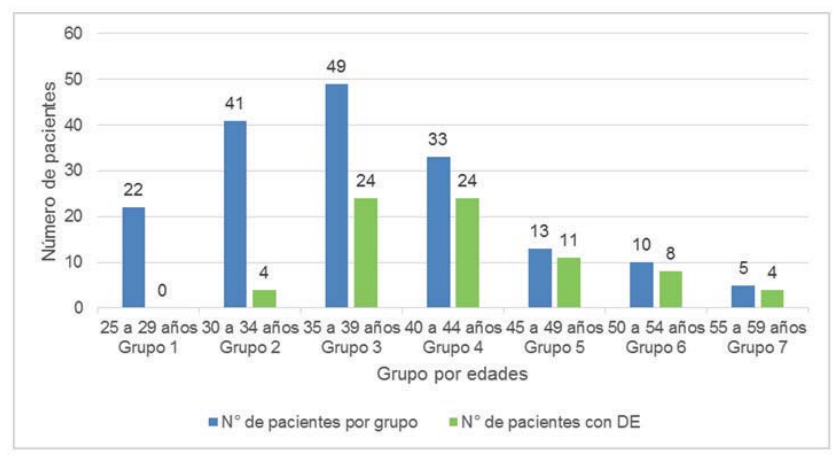

Figura 2. Distribución de la edad y su relación con disfunción endotelial
En el estudio se encontró una proporción menor de pacientes que realizaban más de una hora de ejercicio; de la totalidad de 173 pacientes, el 56\% realizaban ejercicios menos de una hora pero se encontró una relación estadísticamente significativa $(p<0.05)$ al relacionar la presencia de disfunción endotelial con el tiempo realizado, teniendo menor $\mathrm{DE}$ en aquellos que realizaban ejercicios más de una hora, pese a la presencia o no de cualquier otra variable.

La evaluación de regresión nos da además valores estadísticamente significativas, cuando evaluamos de manera conjunta, $(p<0.05)$ siendo esta condición importante en el paciente obeso sea cual sea su nivel de obesidad.

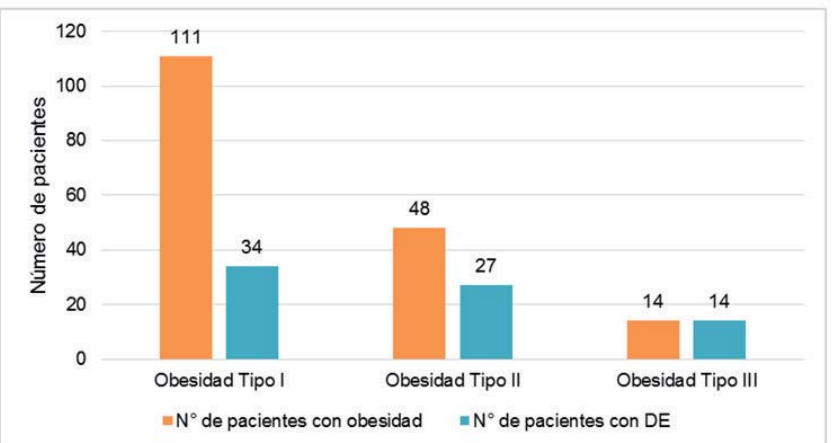

Figura 3. Disfunción endotelial y grado de obesidad

Respecto a la relación entre dieta y $\mathrm{DE}$ se vio una clara relación entre el mayor nivel calórico de dieta y la presencia de disfunción endotelial. De un total de 60 pacientes que consumieron dietas menores de $1800 \mathrm{kcal}$ se encontró la presencia de 14 individuos (23\%) con DE.

En los que presentaron dietas mayores de $1800 \mathrm{kcal}$ que fueron un total de 113 pacientes, se encontró DE en 61 de ellos (54\%), porcentualmente mayor, y que al cruce por método chi-cuadrado fue estadísticamente significativa la relación independientemente de la presencia de otra variable.

En los antecedentes familiares, se encontró la presencia de estos en el $53 \%$ de casos; a su vez, se encontró que hubo una presencia del $65 \%$ de casos con antecedentes familiares en pacientes con DE establecida, sin intervención de otras variables. Se analizó por método de chi-cuadrado, encontrándose 
una relación estadísticamente significativa $(p<0.05)$ Del total de 173 pacientes que conformaron nuestra muestra, 111 pacientes presentaron obesidad tipo I, siendo este el mayor porcentaje.

Así mismo los grupos de obesidad tipo II y III presentaron las mayores tasas porcentuales de disfunción endotelial, siendo el grupo de obesidad tipo III en el que estuvo presente en el $100 \%$ de los individuos evaluados.

Caso contrario pasó con el grupo de obesidad tipo I en el que se vio solo DE en el $30 \%$ de todo el grupo (34 individuos).

Finalmente, hubo presencia de disfunción endotelial en el $43 \%$ de la totalidad de nuestra muestra, situación muy particular con lo que se realizó la prueba de chi-cuadrado, dando un resultado estadísticamente significativo $(p<0.05)$ en el análisis independiente.

Realizando un análisis multivariable de todos los sujetos de nuestro estudio, encontramos una relación estadísticamente significativa $(p<0.05)$ entre el sexo, la edad, la actividad física, los antecedentes familiares y el grado de obesidad, obteniendo como resultado la significación estadística en el cruce de variables.
El único valor que no fue estadísticamente significativo fue la dieta, que al no ser establecida de forma homogénea en la población por causas aj enas al estudio, no se pudo tener una concordancia de resultados, como si lo fue con las otras variables.

\section{DISCUSIÓN}

La enfermedad cardiovascular asociada a la obesidad es un problema que se va conociendo mejor día a día. Con los resultados obtenidos hemos logrado conocer la realidad de la disfunción endotelial en los pacientes con obesidad, con el fin de evaluar la condición de patologías subyacentes que pongan en alto riesgo la vida del paciente $(16,17)$.

En todo esto la consideración de factores de riesgo cardiovascular y las características inherentes de los pacientes tiene una relación directa sobre la evolución y pronóstico del paciente con obesidad (18).

Sobre este punto es que debemos considerar al paciente en múltiples dimensiones tanto en lo físico como en lo funcional, ya que sólo así podremos entender la problemática y el tipo de tratamiento que ofrezcamos en el manejo (19).

Tabla 1. Análisis de regresión multivariable en relación a disfunción endotelial en el personal militar con obesidad

\begin{tabular}{|c|c|c|c|c|c|c|c|}
\hline \multirow{2}{*}{ Modelo } & \multicolumn{2}{|c|}{$\begin{array}{c}\text { Coeficientes no } \\
\text { estandarizados }\end{array}$} & $\begin{array}{c}\text { Coeficientes } \\
\text { estandarizados }\end{array}$ & \multirow{2}{*}{$\mathrm{t}$} & \multirow{2}{*}{ Sig. } & \multicolumn{2}{|c|}{$\begin{array}{c}\text { 25.0\% intervalo de } \\
\text { confianza para B }\end{array}$} \\
\cline { 2 - 4 } & $\mathrm{B}$ & $\begin{array}{c}\text { Error } \\
\text { estándar }\end{array}$ & Beta & & & $\begin{array}{c}\text { Límite } \\
\text { inferior }\end{array}$ & $\begin{array}{c}\text { Límite } \\
\text { superior }\end{array}$ \\
\hline (Constante) & 2.042 & 0.192 & 0.830 & 10648 & 0.000 & 1663 & 2420 \\
Sexo & -0.319 & 0.077 & -0.240 & -4130 & 0.000 & -0.471 & -0.166 \\
Edad & -0.264 & 0.0819 & -0.370 & -5808 & 0.000 & -0.482 & -0.310 \\
$\begin{array}{c}\text { Dieta } \\
\text { Actividad }\end{array}$ & 0.057 & 0.065 & 0.054 & 0.875 & 0.383 & -0.071 & 0.184 \\
Física & -0.464 & 0.059 & -0.464 & -7808 & 0.000 & -0.582 & -0.347 \\
$\begin{array}{c}\text { Antecedentes } \\
\text { Familiares } \\
\text { Grado de }\end{array}$ & -0.210 & 0.047 & -0.221 & 0.750 & 0.000 & -0.320 & 0.120 \\
Obesidad & 0.233 & 0.047 & 0.301 & 4991 & 0.000 & 0.141 & 0.326 \\
\hline
\end{tabular}

a. Variable dependiente: disfunción endotelial

\section{Fuente: Elaboración propia}


Por ej emplo, en nuestro estudio la evaluación de la edad juega un papel importante en la interpretación de resultados para ambos grupos por lo que el factor edad tiene una implicancia como factor independiente para el desarrollo de la disfunción endotelial (20).

Celermajer et al, relacionó la condición de disfunción endotelial con la edad y el género concluyendo una relación directa en donde a más edad, mayor disfunción endotelial (21), esto se comprueba con los hallazgos encontrados en nuestro estudio, donde se manifiesta la misma condición según la disposición por grupo etario (Figura 2).

Otro hallazgo importante es la definición de los grupos por edades basados en la Escala de Framingham (22), la que fue planteada para considerar las condiciones de comorbilidades según edades (intervalos de cinco años) y la asociación de enfermedad cardiovascular como enfermedad coronaria, infartos de miocardio, dislipidemias, entre otras; en la que considerando según esa clasificación, los grupos en hombres antes de los 45 años no presentan un alto nivel de riesgo cardiovascular $(<10 \%)$.

Este punto en particular nos permite estratificar mejor a nuestro paciente obeso de mejor forma, pues hemos encontrado que a pesar de la corta edad de los grupos evaluados, existe una manifestación de la disfuncion endotelial, por lo que debemos establecer rangos diferentes para la evaluación en los programas de manejo de obesidad en la Institución.

Esto se plasma en los resultados obtenidos en donde existe una elevada incidencia de disfunción endotelial por el nivel de obesidad (mayor en la obesidad tipo II y III) que nos hace suponer un incremento en el riesgo real de padecer enfermedades cardiovasculares a temprana edad por esta causa $(23,24)$.

Vemos que en el grupo de sexo masculino, la presencia de obesidad en todos los grupos condiciona la manifestación de disfunción endotelial en el Grupo 2 y 3 de edades, en mucha mayor proporción que en el grupo de mujeres de los mismos grupos. Esto condiciona a pensar que pese a la corta edad de los pacientes, el factor género determina una mayor alteración endotelial y enfermedad cardiovascular de forma precoz. Caso contrario tenemos en el grupo de sexo femenino $(25,26)$.

Esto confirma la investigación de Vilariño et al que evalúa la relación del sexo con la presencia o no de disfunción endotelial, valorando los efectos fisiológicos de las hormonas sexuales, en población sin factores de riesgo, comprobó que las pacientes de sexo femenino presentaron valores superiores en la valoración por vasodilatación mediada por el flujo.

Esta característica la observamos también en nuestras pacientes con obesidad establecida, donde particularmente esa condición las beneficia por el resultado porcentual obtenido de los grupos de menor edad (27-29).

Además, las diferentes respuestas en pacientes sin factores de riesgo cardiovascular podrían deberse al tamaño menor de las arterias, y no a un efecto mediado por las hormonas en sí, situación en particular que no podemos concluir por la presencia de obesidad en toda la muestra $(30,31)$.

Hemos visto la relación directa entre el nivel de actividad física y la presencia o no de la DE. En los criterios de selección para el estudio se consideró esta variable por ser un punto de estudio en muchos trabaj os de investigación.

Se encontró en diversos estudios, la relación positiva en la realización de ejercicio como parte de una mejora en las condiciones de la salud cardiovascular en pacientes con presencia de enfermedad ateroesclerótica, Ilegando a la conclusión que dicha relación tiene una fuerte asociación estadística pues el resultado apunta una menor enfermedad manifiesta clínicamente, además de menor disfunción endotelial evaluada después de un seguimiento a largo plazo (32-34).

A su vez, con los resultados obtenidos vemos que la realización de actividades físicas, que están 
establecidas en la protocolos clínicos de manejo de la obesidad por la CDC: Centers for Disease Control and Prevention y la American Heart Association cumplen con los resultados alcanzados, pues se plantea considerar una actividad física mayor promedio entre 20 y 60 minutos con un plan de ejercicios determinados y en una secuencia progresiva según el nivel de actividad física previa (35-37).

En este punto en particular, en la Institución se cuenta con programassupervisados por entrenadores físicos que brindan un manejo personalizado por la necesidad de recuperar la salud del personal militar en actividad, pero pese a ello el mayor porcentaje de individuos estudiados no puede acceder a este sistema de reentrenamiento por las limitaciones del servicio (distancia de trabajo, horas de labor administrativa, falta de cultura promocional preventiva).

Se observó en el estudio que mientras menor era la edad del paciente y de sexo femenino, mejoraba la tendencia de la práctica de ejercicio por una motivación personal y búsqueda de una mejora física, por lo que en ellos hay la menor tasa de disfunción endotelial asociado a cualquier factor. Caso contrario los de mayor edad tenían muy disminuida la autoestima y el interés por mejorar físicamente.

La significación estadística encontrada entre DE y dieta muestra el grado de relación existente entre ambas, que sin intervenir otra tiene un alto grado de dependencia (38-41).

Pero cabe señalar que en el análisis de regresión, su relación cambió significativamente. Esto nos permite analizar que el tipo de variable dieta en conjunto con el resto, tiene la menor relación estadística respecto al resto de variables; y que este hallazgo es muy importante para nuestro estudio debido a que siendo la variable dieta piedra angular para el tratamiento de la obesidad, no representa necesariamente lo más importante para la condición de disfunción endotelial en obesidad. Considerando los actuales estudios sobre el tema, se considera que los cambios en los etilos de vida y en particular en el tipo de dieta establecida, en donde las dietas hipocalóricas presentan una relación directa con la mejora de la disfuncion endotelial en pacientes seguidos a largo plazo (42).

En nuestra muestra de pacientes, la diversidad de tipos de dietas se clasificó en aquellas mayores de $1800 \mathrm{kcal}$ y en aquellas de menor valor. Eso debido a que se consideró ese valor como un punto de corte para determinar el beneficio de una dieta adecuada para la disminución del peso en pacientes con sobrepeso u obesidad, además del beneficio en reducir las comorbilidades subyacentes en la obesidad como la hipertensión arterial, dislipidemias, diabetes mellitus, entre otras (43).

Por otra parte, dentro de esta división se plantea el tipo de dieta que se debe seguir según las condiciones clínicas del paciente y las metas terapéuticas a alcanzar según el riesgo cardiovascular.

Se plantea establecer dietas con baja cantidad de carbohidratos, muy escasa cantidad de grasa y mucha cantidad de fibras y vegetales, así mismo incrementando al nivel máximo de proteínas $(20 \%$ de la dieta), lo que evidencia una disminución del peso de forma drástica (44).

En nuestro estudio no se pudo considerar esta forma de alimentación por la disposición logística de los comedores en las distintas dependencias de Marina, la facilidad de una asesoría de nutricionistas en el proceso que supervisen la correcta dieta de los pacientes en tratamiento, y sobre todo la falta de voluntad de los pacientes de mantener en todo momento regímenes estrictos en la dieta.

La disminución de calorías básicamente se relacionaba a la menor ingesta del volumen de alimentos pero no a la calidad del mismo. Fueron los grupos de menor edad y mujeres quienes se preocuparon más en recuperar su peso ideal y establecer un régimen dietético estricto, situación que en hombres mayores fue muy difícil de establecer por el programa de nutrición de la Institución.

Considerando estos hallazgos, vemos un mismo 
patrón de conducta por resultados, en donde al realizar la evaluación de la DE en pacientes adultos jóvenes con o sin antecedente de diabetes y otras enfermedades cardiovasculares, encontró un patrón alterado de vasodilatación en aquellos que presentaban dichos antecedentes (45).

Esta característica al margen del grado de obesidad y otras variables, prueba la importancia de establecer un mayor riesgo cardiovascular si los pacientes presentaran dichos antecedentes, habiendo ya un compromiso de la DE en el contexto de obesidad sea el grado menor o mayor.

Por otra parte tenemos que considerar que los pacientes de nuestro estudio que no tenían antecedentes familiares pero presentaron DE, el mayor grupo de ellos fueron de edades mayores, situación que ya hemos analizado antes, donde la edad juega un papel importante para la manifestación de disfunción endotelial de forma independiente.

Con respeto a los resultados, la presencia de disfunción endotelial en relación al tipo de obesidad, es una relación muy directa y característica bajo presencia 0 no de cualquiera de las variables estudiadas.

La distribución de los resultados encontrados nos hace entender más la relación que hay entre el endotelio y la enfermedad cardiovascular, pues vemos que el nivel de disfunción endotelial es una situación que se da en fases tempranas de obesidad, tanto por edad como por nivel de obesidad.

Sabemos que esta alteración además de la presencia de enfermedad ateroesclerótica en el paciente, a causa del aumento de peso, debe considerarse y ser tratada con métodos más complej os y de forma precoz, no sólo pensando en el contexto estético, sino como enfermedad de alto riesgo cardiovascular.

Además la obesidad no sólo afecta a la población adulta, sino a los adolescentes de forma prematura, teniendo en cuenta los mecanismos de acción tanto por inflamación y estrés oxidativo llegando a un daño prematuro de la capacidad regenerativa del sistema cardiovascular.
Llegar a obesidad comprueba según los resultados que la relación de alteración hasta en las mejores condiciones de salud (sólo ser obeso y no tener ninguna enfermedad clásica: HTA, DM2, DLP), tiene ya una asociación directa con el riesgo de alterar fuertemente la salud cardiovascular, por lo que su estratificación de riesgo debe variar, considerándose de muy alto riesgo $(46,47)$.

Los esfuerzos de los distintos programas ofrecidos por la Institución para el control y manejo de la obesidad, aún no son suficientes.

No sólo por la capacidad de atender correctamente a los pacientes con obesidad de una forma más eficiente, sino tomar medidas correctivas de forma primaria, con cambios en los estilos de vida (mayor realización de actividades físicas de entrenamiento, mejor calidad en la alimentación orientado al tipo de labor que efectúe el personal militar en la Institución, a la prevención y manejo del sobrepeso, entre otros) con el fin de evitar el desarrollo de la obesidad precozmente.

La incidencia del control nutricional tiene un punto muy importante en el manejo de la obesidad y la disfunción endotelial como lo hemos demostrado en el estudio, pero más importante aún es el tiempo de actividad física realizada, junto con la presencia de Ios antecedentes, pues estos nos ha dado la mayor influencia en la DE en la condición de obesidad.

Queda mucho por estudiar en este aspecto haciendo comparación en grupos más restrictos de variables y a largo plazo con el fin de evaluar el beneficio de las medidas implementadas en el tratamiento y prevención de la obesidad.

En conclusión, los pacientes obesos evaluados en el presente estudio tienen una alta incidencia de disfuncion endotelial, independientemente de la variable asociada a la que estuviera relacionada, puesto que la obesidad tiene una relación directa con la alteración de la función endotelial, alterando la dinámica del flujo mediado por dilatación, sin la presencia de enfermedad cardiovascular preexistente. 
Existe un factor favorable a la presencia de disfuncion endotelial que es la actividad física, pudiendo tener una menor incidencia de alteración endotelial por ejercicios que contemplen rutinas mayores a una (01) hora, supervisadas por personal calificado.

La dieta, según los resultados del estudio juega un papel importante pero no determinante en la presencia de disfunción endotelial.

De forma secundaria hemos visto que la población obesa de la Institución maneja una condición promedio en la afinidad y adherencia a programas de control de sobrepeso y obesidad, y nutrición, sistema que permite la realización de distintas actividades como el reacondicionamiento físico en pacientes de alto riesgo, sin embargo, vemos una relación inversa a estos programas por parte de la Institución, con el aumento de condiciones laborables sedentarias que no favorecen a las rutinas de ejercicio propuestas.

Por último, se ha demostrado que la evaluación del flujo mediado por dilatación alterado, es un marcador de enfermedad cardiovascular futura, pues se ha logrado reproducir la prueba sin presencia de complicaciones, y confirmando de forma fehaciente los resultados en el estudio, reproduciendo hallazgos similares en otros estudios que evaluaron poblaciones diferentes, por lo que es un procedimiento plausible de ser considerado dentro de las técnicas de selección para control y seguimiento, pero sobretodo estratificación del paciente obeso sin enfermedad cardiovascular por demostrar condiciones técnicas sencillas y su bajo costo de realización. Inclusive poder ser usada para otras enfermedades prevalentes con el fin de estatificar al paciente según su condición endotelial.

\section{Agradecimientos}

A la Oficina del Centro de Investigación de la FMHUNPRG por facilitarnos la relación oficial de tesis y los in extensos no disponibles en la biblioteca.

\section{Presentaciones Previas}

Expuesto en el XXVIII Congreso Científico Nacional de Estudiantes de Medicina Humana.

\section{Fuentes de financiamiento}

Este artículo ha sido financiado por el autor.

\section{Conflictos de interés}

El autor declara no tener conflictos de interés. 


\section{REFERENCIAS BIBLIOGRÁFICAS}

1. Reporte epidemiológico de la Unidad de Evaluación Psicofísica. DICEMENA/DISAMAR MGP 2014. Lima Perú.

2. "BMI Classification". Global Database on Body Mass Index. World Health Organization. 2006. Retrieved July 27, 2012.

3. Palomo G, Fuentes Q, Moore-Carrasc, González D, Rojas A. E consumo de frutas y hortalizas ayuda a prevenir el daño endotelial. Rev Chil Nutr Vol 38, N³, Set 2011

4. Badimon L. Vilahur G. Padro T. Holven KB, Holm T, Nutraceuticals and Atherosclerosis: Human Trials. Cardiovasc Therapeutics 2010; 28: $202-15$

5. Ghisi S, Schenke W, Zalos G, Mincemoyer R, Prasad A. Exercício físico e disfunção endotelial, Arq Bras Cardiol 2010;95(5):e130-e137

6. Bruyndonckx L, Hoymans VY, De Guchtenaere A, Van Helvoirt M, Van Craenenbroeck EM, Diet, exercise and endothelial function in obese adolescents. Pediatrics 2015 Mar, 135(3); e653-61

7. Badimón L, Martínez J. Disfunción endotelial. Rev Esp Cardiol Supl. 2006;6:21A-30A

8. Gori T, Parker JD, Munzel T. Flow-mediated constriction: further insight into a new measure of vascular function. Eur Heart J. 2011; 32:784-7.

9. Toda N, Okamura T. Obesity impairs vasodilatation and blood flow increase mediated by endothelial nitric oxide: an overview J Clin Pharmacol. 2013 Dec;53(12):1228-39

10. Rojas E, Rodríguez-Molina D, Bolli P. The role of adiponectin in endothelial dysfunction and hypertension. Curr Hypertens Rep. 2014 Aug;16(8):463

11. Prieto D, Contreras C, Sánchez A. Endothelial dysfunction, obesity and insulin resistance. Curr Vasc Pharmacol. 2014 May;12(3):412-26.

12. Lovren F, Teoh H, Verma S. Obesity and Atherosclerosis: Mechanistic Insights. Canadian Journal of Cardiology. 2015 Feb; 31(2):177-183.

13. Thijssen DHJ, Black MA, Pyke KE, Padilla J, Atkinson G, Harris RA, et al. Assessmentof flow-mediated dilation in humans: a methodological and physiological guideline. Am J Physiol Heart Circ Physiol. 2011;

14. Antonio L. Arrebola-Moreno, Martin Laclaustra y Juan Carlos Kaskia. Evaluación no invasiva de la función endotelial en la práctica clínica. Rev Esp Cardiol. 2012;65(1):80-90

15. Charakida M, Masi S, Lu“ scher TF, Kastelein JJP, Deanfield JE. Assessment ofatherosclerosis: the role of flow-mediated dilatation. Eur Heart J. 2010; 31:2854-61.

16. World Health Organization Technical report series 894: "Obesity: preventing and managing the global epidemic." Geneva: World Health Organization, 2000. PDF. ISBN 92-4-120894-5.

17. Iantorno $M$, Campia $U$ Obesity, inflammation and endothelial dysfunction. J Biol Regul Homeost Agents. 2014Apr-Jun;28(2):169-76

18. Obesidad y Sobrepeso, Nota descriptiva N`311 WHO, 2015-01

19. Cardillo C. Drug treatments to restore vascular function and diabesity. Ann Pharm Fr. 2013 Jan;71(1):27-33
20. Tisha Marie B. Suboc, Kodlipet Dharmashankar. Moderate obesity and endothelial dysfunction in humans: influence of gender and systemic inflammation Physiological Reports2013. Vol. 1. Iss. 3

21. Celermajer DS, Sorensen KE, Gooch VM, Spiegelhalter DJ, Miller OI, Sullivan ID,et al. Non-invasive detection of endothelial dysfunction in children and adultsat risk of atherosclerosis. Lancet. 1992

22. You T, Arsenis NC, Disanzo BL, Lamonte MJ Effects of exercise training on chronic inflammation in obesity : current evidence and potential mechanisms. Sports Med. 2013 Apr; 43(4):243-56.

23. Tisha Marie B. Suboc, Kodlipet Dharmashankar. Moderate obesity and endothelial dysfunction in humans: influence of gender and systemic inflammation Physiological Reports2013. Vol. 1. Iss. 3

24. Gu P, Xu A. Interplay between adipose tissue and blood vessels in obesity and vascular dysfunction. Rev Endocr Metab Disord. 2013 Mar;14(1):49-58.

25. Kim J, Montagnani M, Chandrasekran S, Quon MJ Role of lipotoxicity in endothelial dysfunction. Heart Fail Clin. 2012 Oct;8 (4):589-607.

26. Seligman, B.G., Polanczyk, C.A., Santos, A.S., Foppa, M., Junges, M., Bonzanini, L., Intensive practical lifestyle intervention improves endothelial function in metabolic syndrome independent of weight loss: a randomized controlled trial. Metabolism: Clin. Exp. 2011; 60:1736-1740.

27. Mohler, E.R. 3rd, Sibley, A.A., Stein, R., Davila-Roman, V., Wyatt, H., Badellino, K., Endothelial function and weight loss: comparison of low-carbohydrate and low-fat diets. Obes. (Silver Spring, Md). 2013; 21:504-509.

28. Meyers MR, Gokce N. Endothelial dysfunction in obesity: etiological role in atherosclerosis. Curr Opin Endocrinol Diabetes Obesity. 2007 Oct;14(5):365-9

29. Michael D. Jensen, Donna H. Ryan, Caroline M. Apovian, Jamy D. Ard. 2013 AHA/ACC/TOS Guideline for the Management of Overweight and Obesity in Adults: A Report of the American College of Cardiology/American Heart Association Task Force. Circulation. published online November 12, 2013

30. Phillips SA, Mahmoud AM, Brown MD, Haus JM. Exercise interventions and peripheral arterial function: implications for cardio-metabolic disease. Prog Cardiovasc Dis. 2015 Mar-Apr;57(5):521-34

31. Joris PJ, Zeegers MP, Mensink RP. Weight loss improves fasting flowmediated vasodilation in adults: A meta-analysis of intervention studies. Atherosclerosis. 2015 Mar; 239(1):21-30

32. Hallmark R, Patrie JT, Liu Z, Gaesser GA, Barrett EJ, Weltman A.The effect of exercise intensity on endothelial function in physically inactive lean and obese adults PLoS One. 2014 Jan 20;9

33. Ades, P.A., Savage, P.D., Lischke, S., Toth, M.J., Harvey-Berino, J. Bunn, J.Y., The effect of weight loss and exercise training on flowmediated dilatation in coronary heart disease: a randomized trial. Chest.2011; 140: 1420-1427.

34. Green, D.J., Maiorana, A., O'Driscoll, G., Taylor, R. Effect of exercise training on endothelium-derived nitric oxide function in humans. J. Physiol. 2004; 561:1-25.

35. Dod, H.S., Bhardwaj, R., Sajja, V., Weidner, G., Hobbs, G.R., Konat, G.W. et al, Effect of intensive lifestyle changes on endothelial function and on inflammatory markers of atherosclerosis. Am. J. Cardiol. 2010;105:362-367 
36. Montero, D., Walther, G., Perez-Martin, A., Roche, E., Vinet, A. Endothelial dysfunction, inflammation, and oxidative stress in obese children and adolescents: markers and effect of lifestyle intervention. Obes. Rev. Off. J. Int. Assoc. Study Obes. 2012;1 3:441-455.

37. Andrés $\mathrm{E}$, León $\mathrm{M}$, Cordero A, Magallón R. Factores de riesgo cardiovascular y estilo de vida asociados a la aparición prematura de infarto agudo de miocardio Rev Esp Cardiol. 2011; 64:527-9. Vol. 64 Núm.06

38. Sankhla M., Sharma T.K., Mathur K., Rathor J.S., Butolia V., Relationship of oxidative stress with obesity and its role in obesity induced metabolic syndrome. Clin. Lab. 2012 ;58:385-392

39. Thomazella, M.C., Goes, M.F., Andrade, C.R., Debbas, V., Barbeiro, D.F., Correia, R.L. Effects of high adherence to mediterranean or low-fat diets in medicated secondary prevention patients. Am. J. Cardiol.2011;108:1523-1529

40. Buscemi, S., Cosentino, L., Rosafio, G., Morgana, M., Mattina, A., Sprini, D. Effects of hypocaloric diets with different glycemic indexes on endothelial function and glycemic variability in overweight and in obese adult patients at increased cardiovascular risk. Clin. Nutr. Edinb. Scotl. 2013; 32:346-352.

41. Nagamia, S., Pandian, A., Cheema, F., Natarajan, R., Khan, Q.A., Patel, A.D.I, The role of quinapril in the presence of a weight loss regimen: endothelial function and markers of obesity in patients with the metabolic syndrome. Prev. Cardiol. 2007; 10:204-209.

42. Tuan M, Pham I, Valensi P, Rousseau H. Flow-mediated-paradoxical vasoconstriction is independently associated with asymptomatic myocardial ischemia and coronary artery disease in type 2 diabetic patients. Cardiovascular Diabetology 2014, 13:20

43. Lee HY, Sakuma I, Ihm SH, Goh CW, Koh KK. Statins and reninangiotensin system inhibitor combination treatment to prevent cardiovascular disease Circ J. 2014;78 (2):281-7.

44. Angelico, F., Loffredo, L., Pignatelli, P., Augelletti, T., Carnevale, R., Pacella, A., Weight loss is associated with improved endothelial dysfunction via NOX2-generated oxidative stress down-regulation in patients with the metabolic syndrome. Intern. Emerg. Med. 2012; 7:219-227.

45. Bigornia, S.J., Mott, M.M., Hess, D.T., Apovian, C.M., McDonnell, M.E., Duess, M.A., Long-term successful weight loss improves vascular endothelial function in severely obese individuals. Obes. (Silver Spring, Md).2010; 18:754-759.

46. Ras, R.T., Streppel, M.T., Draijer, R., Zock, P.L. Flow-mediated dilation and cardiovascular risk prediction: a systematic review with meta-analysis. Int. J. Cardiol. 2013; 168:344-351.

47. Gu, P., Xu, A.M. Interplay between adipose tissue and blood vessels in obesity and vascular dysfunction. Rev. Endocr. Metab. Dis. 2013; 14:49-58.

\section{Correspondencia:}

Oscar Luis Alvarez Franco

Dirección: Av. El Corregidor 1531, La Molina, Lima - Perú

Teléfono: $365-2300$

Correo electrónico: oscaralvarezmd@gmail.com 